Publié :

Mathieu Gervais, « Le paysan dans l'imaginaire écologique :

l'utopie rustique d'Henri Mendras », Revue du MAUSS, 2/2013 (n 42), p. 339-353. 


\title{
Le paysan dans l'imaginaire écologique : l'utopie rustique d'Henri Mendras
}

\author{
Mathieu Gervais
}

Souvent pensée de l'intérieur comme un mouvement utopique, l'écologie politique a parfois été pensée de l'extérieur comme faisant le jeu de l'idéologie dominante, c'est-à-dire du capitalisme. Ainsi, on trouve d'un côté, et dès le début du mouvement écologiste en France, des penseurs qui inscrivent clairement leur pensée dans un schéma de subversion de l'idéologie. Serge Moscovici, par exemple, place l'écologie dans une « histoire hétérodoxe ${ }^{1}$ » qui reprend sciemment une généalogie déjà dépeinte par Ernst Bloch². Ici, l'écologie entend bien mettre en œuvre de manière programmatique une « conscience qui ne coüncide pas avec l'“être" à ses alentours », soit une conscience utopique au sens de Karl Mannheim³ . D'un autre côté, une tradition d'étude sociologique de l'écologie met en lumière l'étroitesse de sa base sociale, regroupée autour des classes moyennes intellectuelles ${ }^{4}$, et analyse l'engagement écologiste en ayant recours à l'idée de lutte contre le déclassement ${ }^{5}$. Ces

1. Serge Moscovici, Hommes domestiques et hommes sauvgaes, UGE, Paris, 1974.

2. Ernst Bloch, Thomas Münzer, UGE, Paris, 1964, p. 305.

3. Karl Mannheim, Idéologie et utopie, Maison des sciences de l'homme, Paris, 2006, p. 159.

4. Daniel Boy, «Le vote écologiste : évolutions et structures », Cahiers $d u$ CEVIPOF, $\mathrm{n}^{\circ}$ 6, février 1991.

5. Bernard Lacroix, L'Utopie communautaire, PUF, Paris, 1981 ; et Danièle Léger, Bertrand Hervieu, Des Communautés pour les temps difficiles, Le Centurion, Paris, 1983. 
analystes proposent parfois de voir là l'indice d'une relégation des clivages économiques au profit des clivages « postmatérialistes ${ }^{6} »$, relégation qui atténuerait les critiques à l'encontre du capitalisme ${ }^{7}$.

Afin d'étayer ce débat, nous voulons proposer sa mise en abîme à travers une réflexion autour d'une œuvre littéraire utopique mettant en scène des idéaux écologiques. Il s'agit du Voyage au pays de l'utopie rustique ${ }^{8}$, écrit en 1979 par Henri Mendras, chef de fil de la sociologie rurale en France. Choisir cette étude revient à déplacer notre définition de l'utopie afin d'emprunter aussi des critères issus des études littéraires. Le texte d'Henri Mendras est ainsi considéré comme s'inscrivant dans un genre littéraire précis obéissant à un certain nombre de règles fixes ${ }^{9}$. Il combine un récit de voyage fictif - celui d'Alexis, diplomate d'une république soviétique imaginaire d'Asie centrale, le Khoragstan - avec la description de l'état parfait ${ }^{10}$ - ici le Pays de l'Utopie Rustique (PUR), localisé dans certaines régions de France ${ }^{11}$. Ce détour par la littérature utopique au sein de l'imaginaire écologique doit donc nous permettre d'interroger l'ambiguité sémantique propre de l'utopie : entre irréalité (ou-topos) et idéalité (eu-topos) $)^{12}$. Quelles sont les « images-souhaits » (Ernst Bloch) dessinées par ce récit ? Surtout que révèle de l'écologie son inscription dans une généalogie utopique : quelle sont les continuités et les ruptures entre ce récit et des récits utopiques antérieures?

6. Robert Inglehart, La Transition culturelle dans les sociétés industrielles avancées, Économica, Paris, 1993.

7. Hubert Billemont, dans sa thèse sur L'Écologie politique : une idéologie de classes moyennes, parle à propos de la pensée écologiste d' " un anti-ouvriérisme larvé et [d']un anti-capitalisme bien pensant et humanisant (c'est-à-dire éloigné de l'orthodoxie communiste)» (thèse de sociologie non publiée, 2006, p. 50).

8. Henri Mendras, Voyage au pays de l'utopie rustique, Actes-Sud, Arles, 1992.

9. Jean-Michel Racault, Nulle part et ses environs, PUPS, Paris, 2003, p. 13.

10. Alberto Petrucciani, « La déconstruction du discours utopique entre le xixe et le xxe siècle », in Heinrich Hudde et Peter Kuon (dir.), De l'utopie à l'uchronie : formes, significations, fonctions, Gunter Narr Verlag, Tübingen, 1988 (actes d'un colloque de 1986 à Erlangen), p. 135.

11. Principalement le Massif central et ses pourtours, le grand Sud-Ouest, et la Bretagne.

12. Jean-Michel Racault, op. cit., p. 15. 


\section{Une critique interne de l'utopie}

Le voyage au pays de l'utopie rustique s'ouvre par la lettre de mission adressée au « camarade Alexis » (protagoniste et narrateur du récit) par le comité central du Khoragstan. Cette mission consiste à effectuer dans la France de 2007 (rappelons que Mendras écrit en 1979) un voyage d'étude de l'utopie rustique. Ce voyage s'inscrit dans la réflexion du pays quant à son avenir : quel modèle embrasser ? Trois modèles sont alors évoqués comme repoussoir : le modèle occidental - lié à l'industrialisation et à la suprématie technique mais aussi à la pauvreté et à la pollution -, le modèle russe - lié aussi à l'industrie mais sous le contrôle d'une « dictature policière » et le modèle chinois - lié à la « toute puissance du parti et de l'armée ${ }^{13} »$.

Cette préface annonce le ton du roman : il ne pourra s'agir que d'une utopie construite dans un rapport critique à un idéal industriel et technologique, si puissant dans les projections imaginaires depuis le $\mathrm{XIX}^{\mathrm{e}}$ siècle $^{14}$, qu'il se décline politiquement dans la démocratie occidentale ou dans le soviétisme russe ${ }^{15}$. De plus, on trouve un positionnement précis par rapport au communisme en tant qu'utopie dominante. La cité idéale proposée par le communisme est d'emblée dépréciée dans la confrontation à sa réalité politique (en Russie et en Chine) qui la rapproche de manière négative de la cité idéale capitaliste (industrie et technique) et de la cité idéale conservatrice (qui refreine l'émancipation individuelle). Il y a donc une mise à distance du communisme mais au sein même d'une culture socialiste ${ }^{16}$, ce qui correspond à une « critique interne de l'utopie ${ }^{17} »$.

L'originalité de cette approche inscrit, selon nous, ce texte de Mendras dans une lignée d'utopies qui part de William Morris (Nouvelles de nulle part, 1890) et passe par Alexandre Chayanov (Voyage de mon frère Alexis au pays de l'utopie paysanne, 1920,

13. Henri Mendras, Voyage au pays de l'utopie rustique, op. cit., p. 8-9.

14. Voir par exemple la manière dont la vie s'organise autour du travail industriel et rationalisé chez Robert Owen, Charles Fourier ou Étienne Cabet.

15. Henri Mendras, ibid., p. 9.

16. Ibid., p. 7-12.

17. Dayan-Herzbrun Sonia et al., «L'homme est un animal utopique : entretien avec Miguel Abensour », Mouvements, $\mathrm{n}^{\circ}$ 45-46, 2006/3, p. 81. 
écrit sous le pseudonyme d'Ivan Kremniov) $)^{18}$. De ces deux auteurs, Mendras distingue surtout le second, à qui il reprendra le concept d'économie paysanne dans son œuvre de sociologue et à qui son utopie doit beaucoup ${ }^{19}$. Déjà, chez Morris et Chayanov, le problème est celui de l'utopie après l'utopie communiste. Chez eux, le ressort narratif se déploie sous la forme d'un rêveur qui pénètre dans le monde utopique. Les protagonistes y sont des cadres socialistes qui s'endorment en songeant aux limites de la Révolution et se réveillent dans un monde utopique qui transcende leurs doutes ${ }^{20}$.

On distingue alors une progression entre ces trois livres. De Morris à Mendras, la critique de l'utopie communiste s'émancipe de plus en plus du cadre imaginaire socialiste. Mendras n'est ni lui-même militant comme Morris, ni citoyen d'une république soviétique comme Chayanov. Son protagoniste vient, certes, d'une république socialiste, mais il ne rêve pas d'une utopie postcommuniste, il visite une utopie rustique.

\section{Entre l'ici et l'ailleurs}

Le livre de Mendras, comme ceux de Morris et de Chayanov avant lui, illustre une évolution dans la forme des récits utopiques : le passage d'un ailleurs géographique à un ailleurs temporel :

« À travers le renvoi, explicite ou chiffré, entre l'ailleurs et le hic et nunc, le jeu des duplications, l'utopie vient s'insérer comme un "pouvoir-être-autrement", comme possibilité active ( $c f$. Bloch). La transposition spatiale, cependant, cristallise dans le temps la possibilité, le temps futur, en donnant une image synchronique et même bloquée, immobile ${ }^{21}$.»

D'un point de vue littéraire donc, la transformation de l'ailleurs utopique peut être interprétée comme le passage à l'utopie en tant que possibilité, en tant que tension efficace vers l'avenir. Il ne

18. Nous isolons ces deux références dans un souci d'illustration et non d'exhaustivité : William Morris, Nouvelle de nulle part ou une ère de repos, L'Altiplano, Montreuil, 2009 ; Ivan Kremniov (Alexandre Chayanov), Voyage de mon frère Alexis au pays de l'utopie paysanne, L'Âge de l'homme, Lausanne, 1976.

19. Le livre de Chayanov est d'ailleurs directement cité : Henri Mendras, op. cit., p. 106.

20. William Morris, op. cit., p. 9; et Ivan Kremniov, op. cit., p. 12.

21. Alberto Petrucciani, op. cit., p. 136. 
s'agit plus d'un état irréel mais bien d'un état idéal. Chayanov qui regarde Moscou par la fenêtre, Mendras qui marche rue Mouffetard à Paris, tous les deux imaginent in situ ce que pourraient être ces endroits si familiers : Moscou transformé en ville moyenne, bourg campagnard $^{22}$; la rue « Mouffe » devenue lieu de convivialité, ses rues rendues aux passants et à la végétation ${ }^{23}$. Surtout, depuis la balade sur la Tamise du protagoniste des Nouvelles de nulle part, ces auteurs mettent en œuvre une nouvelle façon de voyager en utopie. Il ne s'agit pas d'épuiser les arcanes juridico-politiques ${ }^{24}$ d'une cité idéale mais plutôt d'apprécier l'effet sur les sens, la différence qualitative que recouvre cet ailleurs si familier : l'air sain de Londres et l'eau propre de la Tamise chez Morris, la qualité de l'art dans les campagnes russes chez Chayanov, les promenades en forêt à Florac ou en Haute-Garonne chez Mendras... Et les trois auteurs se retrouvent encore pour insister sur la qualité de la nourriture dans leurs utopies respectives. Morris et Chayanov prennent même le temps de faire flirter leurs personnages avec les femmes qui leur servent de guide. Selon Miguel Abensour, depuis Morris advient « une nouvelle qualité, celle du "merveilleux utopique" » :

« Le merveilleux qui selon Mabille "veut dépasser les limites de l'espace et du temps, veut détruire les barrières, il est la lutte de la liberté contre ce qui la réduit, la détruit, la mutile... il est tension passionnelle et poétique". William Morris joue du merveilleux contre la clôture du modèle juridico-politique. Il crée ainsi une nouvelle forme d'utopie, résolument moderne en ce qu'elle est expérimentale, œuvre ouverte, inachevée ${ }^{25}$. 》

Avec ces partis-pris narratifs, on peut se trouver frustré car « tout ceci apparaît donc parfaitement subjectif, sélectif, partiel et partial $^{26} »$. Cependant, on peut encore y voir le dépassement de l'utopie, sa critique interne. En effet, les auteurs font le pari

22. Kremniov, op. cit., p. 26.

23. Henri Mendras, op. cit., p. 19.

24. Ces arcanes sont aussi évoquées mais le plus souvent sous la forme de discussions avec des référents intellectuels : un vieillard de cent cinq ans, responsable de la bibliothèque du British Museum chez Morris, un « vieux sociologue » chez Mendras. Chez Chayanov, ce récit central d'éclaircissement vient de la lecture d'un livre d'histoire.

25. Dayan-Herzbrun Sonia et al., op. cit., p. 81.

26. Armand Frémond, « Voyage au pays de l'utopie rustique », Espace géographique, $\mathrm{n}^{\circ} 10,1981$, p. 48. 
de la subjectivité comme gage de réalisme : les descriptions sont subjectives et partielles car l'état utopique nous échappe par définition. Il est fait pour ceux qui y vivent et non pour nous ${ }^{27}$. L'auteur abandonne alors sa toute-puissance au profit de la vraisemblance de l'idéal qui nécessite l'inachèvement et l'ouverture de l'utopie ${ }^{28}$. Il n'est plus démiurge, il se fait voyageur dans des horizons ouverts par son propre rêve mais qui le dépassent. À travers son narrateur, il envoie une sonde qui nous renseigne sur la profondeur de l'utopie mais non sur son étendue.

L'image-souhait se dote donc d'une épaisseur sensuelle qui la rend proche, palpable, voire envisageable : les lieux sont connus, leur qualité seule change. Toutefois, si sous l'angle sensuel l'utopie devient envisageable, elle reste politiquement lointaine chez Morris et Chayanov : les narrateurs rêvent, l'utopie intervient comme une parenthèse dans leurs biographies respectives mais aussi dans l'ordre historique. Ils effectuent par le rêve un saut dans le temps, qui les projette au-dessus de développements historiques complexes rapidement brossés qui mettent en jeu guerres et révolutions. Au contraire, chez Henri Mendras, nous sommes conduits à nous identifier à Alexis, voyageur contemporain de l'utopie, qui y accède non par hasard mais par sa propre volonté. Les protagonistes de Morris et Chayanov débutent leurs voyages par un réveil déroutant en pays inconnu, tandis que celui de Mendras descend d'un avion à l'aéroport de Paris, ville de sa jeunesse étudiante. L'ailleurs de l'utopie est ici doublement proche : il n'est pas dans un lieu inaccessible et inconnu, il n'est pas non plus dans un futur lointain.

Loin des guerres mondiales et des révolutions sanglantes de Morris et Chayanov, le PUR (pays de l'utopie rustique) de Mendras s'est développé dans un pays riche - à l'image de la France des années 1970 - et grâce à une importante mobilisation politique née de «la conjonction de [la] Nouvelle politique agricole et des mouvements régionalistes, écologiques et pédagogiques ${ }^{29} »$. L'auteur insiste surtout sur la dimension pédagogique de l'évolution, qualifiée de « révolution culturelle ${ }^{30} »$, et qui correspond à la mise

27. Alberto Petrucciani, op. cit., p. 140.

28. Ibid., p. 139.

29. Henri Mendras, op. cit., p. 94.

30. Ibid., p. 95. 
en œuvre d'une nouvelle pédagogie dans un ensemble d'écoles situées en milieu rural.

On retrouve, dans cet optimisme, l'atmosphère caractéristique de la lutte écologique des années 1970. Comme dans l'An 01 31, il suffit d'un pas de côté pour que le système se réforme. La seule volonté d'une partie mobilisée de la population transforme la vie de manière décisive ${ }^{32}$. La richesse étant disponible, il suffit de la transférer et de la compléter par des activités gratuites pour atteindre l'idéal social recherché. Or le régime démocratique permet le déroulement pacifique de ces transferts, d'où l'aspect paisible de cette révolution. Plus que jamais, l'espace entre l'ici et l'ailleurs utopique semble donc ouvert et accessible ; et si elle se détourne radicalement du cours normal de l'histoire, l'utopie de Mendras puise dans la société présente les conditions de sa réalisation.

\section{La ville à la campagne}

Chez Mendras, cette société utopique où la qualité de la vie est optimale prend, comme chez Chayanov, la forme d'une société « rustique » ou « paysanne ». Cela signifie d'abord une inversion de la répartition de la population entre ville et campagne, thème déjà présent chez Morris ${ }^{33}$. Chayanov parle d'un " décret sur la destruction des villes de plus de vingt mille habitants ${ }^{34} »$ qui a vidé Moscou de sa population ${ }^{35}$. À l'inverse, il décrit des champs « où des milliers de paysans » travaillent ${ }^{36}$. Pas de compte chez Mendras, mais quelques indications allant dans le même sens : les HLM des banlieues tombent en ruine ${ }^{37}$ et les hameaux ruraux se repeuplent. De plus, cette vitalité démographique des campagnes est incarnée par deux exemples types dans le récit de Mendras : l'urbain s'installant

31. Gébé, L'An 01, Le Square, Paris, 1972.

32. Henri Mendras, op. cit., p. 95.

33. Serge Latouche, « Préface », in William Morris, Comment nous pourrions vivre, éd. Le Passager clandestin, Neuvy-en-Champagne, 2010.

34. Kremniov, op. cit., p. 26 et 31.

35. Moscou comptait environ un million d'habitants en 1920, le Moscou utopique de 1984 en compte cent mille.

36. Ibid., p. 41.

37. Henri Mendras, op. cit., p. 94. 
agriculteur, qu'il soit originaire de la région ou non ${ }^{38}$, et les retraités qui retournent dans le village de leur enfance ou qui viennent s'installer près des écoles nouvelles afin de pouvoir s'occuper de leur petits-enfants ${ }^{39}$. Au final, les campagnes ont retrouvé, nous dit l'auteur, « la population d'il y a un siècle et demi », c'est-à-dire de 1857, époque historique du peuplement maximal de la campagne française ${ }^{40}$. Cette ruralisation de la société prend l'aspect de «la ville à la campagne ${ }^{41}$ », qui réconcilie de manière concrète culture et nature. En effet, pour Mendras après Chayanov, la densification des campagnes entraîne la multiplication des services et des activités. De fait, la vitalité des communautés villageoises, les services culturels et les avancés techniques ${ }^{42}$ (télétravail et magnétoscope) désenclavent complètement les ruraux.

\section{L'économie paysanne}

Cette structure spatiale et culturelle de l'utopie repose sur une façon neuve d'appréhender l'économie, organisée autour du concept d'économie paysanne. Idée sociologique clé des travaux scientifique de Chayanov puis de Mendras, l'économie paysanne recouvre le fonctionnement économique traditionnel du foyer paysan qui ne serait pas saisissable dans les termes économiques classiques. Le constat à l'origine de ce concept est l'absence de capital et de salaire au sein d'une ferme traditionnelle : le travail est réparti en fonction des besoins de la famille (« équilibre travail-consommation ») et les produits du travail sont principalement destinés à l'autoconsommation. Il n'y a pas de calcul capitaliste pour maximiser les profits mais plutôt un calcul subjectif pour maintenir un niveau de vie constant où l'équilibre entre pénibilité du travail et satisfaction des besoins est optimal. Cette satisfaction des besoins passe d'abord par l'autoconsommation mais se prolonge dans l'échange marginal avec

38.Ibid., p. 67 et p. 26.

39.Ibid., p. 171.

40. Selon Jacques Dupaquier (Histoire de la population française, PUF, Paris, 1988), la France des années 1850 comptait presque trente millions de ruraux, soit trois fois plus qu'en 2011.

41. Henri Mendras, op. cit., p. 41.

42. Ibid., p. 79. 
l'extérieur : vente d'une part de la production mais aussi vente de la force de travail, ou activité complémentaire à l'activité agricole ${ }^{43}$.

Les Cévenols de Mendras sont des néoruraux heureux parce qu'ils ont retrouvé le principe de l'économie paysanne : "Des cultures vivrières pour notre consommation et une activité artisanale pour l'argent ${ }^{44}$. » En opposition avec l'économie de marché, la question de la rentabilité au sens strict n'a plus de sens ici car l'autoconsommation est la norme, le travail salarié l'exception, les échanges de services entre voisins évidents...

Pour Mendras, la généralisation de cette économie est rendue possible par la «moyennisation de la sociétét ${ }^{45} »$, soit l'enrichissement de la société et la constitution d'une vaste classe moyenne dont la demande augmente qualitativement et non plus quantitativement. De plus, pour lui, c'est la généralisation des congélateurs qui permet la diffusion de ce mode de consommation en le rendant accessible aux urbains qui viennent s'approvisionner à la campagne ${ }^{46}$. L'agriculture se voit érigée en «mode de vie ${ }^{47}$ » fondé sur une production agricole de qualité et intensive en main-d'œuvre mais qui va bien au-delà, donnant son ossature économique à la société tout entière tournée vers l'autoconsommation.

\section{Devenir paysan : de l'importance de la pédagogie}

Si chez Chayanov le principe de l'économie paysanne était déjà présent, il résultait non d'une refondation mais d'une généralisation - fruit de la prise de pouvoir de la paysannerie - d'un principe encore vivant dans la Russie de $1920^{48}$. À l'inverse, l'œuvre de Mendras s'inscrit dans une époque marquée par la Fin des pay-

43. Alexandre Chayanov, The Theory of Peasant Economy, American economic association, Homewood, 1966 ; Henri Mendras, Les Sociétés paysannes, Gallimard, Paris, 1995.

44. Henri Mendras, op. cit., p. 27.

45. Henri Mendras, La Seconde Révolution française. 1965-1984, Gallimard, Paris, 1988.

46. Henri Mendras, Voyage au pays de l'utopie rustique, Actes Sud, Arles, 1992, p. 93.

47. Ibid., p. 87.

48. Ivan Kremniov, op. cit., p. 50. 
sans $^{49}$. De ce fait, toute son utopie est traversée de la question de la renaissance d'une société rurale disparue. La pédagogie et les enfants occupent alors une place centrale dans le livre et dans le PUR. Le changement de mode vie induit par le PUR met en effet en branle un retour à une relation particulière à l'espace, au " pays » en tant que lieu de vie et de travail mais aussi d'identification culturelle qui s'oppose au mode de vie urbain et industrialisé. Ainsi, ce nouveau mode de vie "surprend chez des adultes, élevés en ville et venus tardivement à la campagne ». Au contraire, chez les enfants, il « paraît naturel [...]. Pour eux, le PUR est un véritable paradis $^{50} \gg$. Dans la pédagogie également, il s'agit de réconcilier culture et nature sous la forme d'une conciliation entre savoir-faire et connaissances, technique et science, travail et réflexion. Fidèle à cette perspective, le système éducatif dépeint par Mendras comprend plusieurs principes : encouragement de l'initiative, de l'autonomie et de la responsabilité ; alternance entre des stages pratiques et des temps à l'école ; temps scolaire réduit et participation aux tâches de la communauté ${ }^{51}$. Au lycée, sont enseignées cinq matières principales : «Le latin, les mathématiques, l'ethnologie, l'art et la forêt $^{52} »$. Ce programme éducatif et la vitalité des enfants du PUR produisent un vif effet sur le narrateur, qui voit chez eux « toutes les qualités humaines de nos jeunes nomades du Nord doublées du raffinement d'une des plus vieilles civilisations ${ }^{53} »$, description qui n'est pas sans nous rappeler l'Émile de Jean-Jacques Rousseau, qui lui aussi réconciliait culture et nature, en étant un « sauvage fait pour habiter les villes ${ }^{54} \gg$.

\section{Le passé : la famille}

À travers cette question de la ré-invention du paysan, celui-ci entendu au sens premier d'habitant du pays et pas seulement en tant

49. Henri Mendras, La Fin des paysans, Actes Sud, Arles, 1992 (1967).

50. Henri Mendras, Voyage au pays de l'utopie rustique, op. cit., p. 29.

51. Ibid., p. 49-52.

52. Ibid., p. 37.

53. Ibid., p. 58.

54. Jean-Jacques Rousseau, Émile ou De l'éducation (1762), Les classiques des sciences sociales, Chicoutimi (Québec), 2002, III, p. 159. 
qu'agriculteur, on perçoit un enjeu utopique propre : la question de ce qu'il faut reprendre du passé.

Économiquement, nous avons brièvement vu ce que l'économie du PUR devait à la tradition paysanne. Un autre exemple significatif est la famille, à laquelle Mendras, après Chayanov, accorde également une place prépondérante. Ces auteurs dressent en détail le portrait de deux grandes familles paysannes nombreuses et enracinées dans leurs terroirs et qui incarnent l'unité de base de l'utopie : les Minine chez Chayanov, les Astruc chez Mendras ${ }^{55}$.

À côté de ce modèle familial traditionnel, Alexis se confronte à un autre, plus moderne, lorsqu'il visite le lycée forestier de Saint-Bertrand-de-Comminges, en Haute-Garonne : la famille Gellenave ${ }^{56}$. Cette famille est bâtie autour de la grand-mère, qui s'est mariée trois fois, qui a une fille et un garçon (de deux pères différents). Aujourd'hui à la retraite, elle s'est installée près du lycée de sa petite fille de quinze ans qui habite donc chez elle. Sa fille ne s'est jamais mariée. Mendras évoque, au fil du livre, la généralisation de ces modèles familiaux centrés sur les grandsparents : la libéralisation des unions (divorces, concubinages) et l'allongement de l'espérance de vie contribuent au regroupement de la famille autour du lieu d'habitation des grands-parents :
«C'est une variante du modèle Astruc, sans aucune origine paysanne ni dimension agricole : ce sont des urbains, de professions diverses, qui ont spontanément reconstitué le parentage villageois que décrivent historiens et ethnologues. Et ce parentage vous pouvez y vivre au centre, en marge, ou vous en isoler s'il vous pèse ${ }^{57}$. »

Finalement, la famille paraît structurellement centrale dans l'argumentaire de Mendras :

«Sans la force de la famille, nous n'aurions pas pu développer le PUR car [...] le PUR est fondé sur la famille et réciproquement le PUR a renforcé la famille, tout en recréant la communauté locale ${ }^{58}$. »

Fasciné par l'ethnologie, Mendras semble concevoir la famille comme la structure indispensable à l'ancrage de l'individu dans la société. La famille permet la société, particulièrement en France,

55. Ivan Kremniov, op. cit., p.42 et Henri Mendras, op. cit., p. 61 et p. 69-71.

56. Ibid., p. 172.

57. Ibid., p. 102.

58. Ibid., p. 100. 
si on en croit ses analyses ${ }^{59}$. Mais là aussi, comme pour la question du paysan, il s'agit de refaire famille, de distinguer dans le passé le modèle communautaire fondé sur la famille et de le prolonger dans le présent. Cette reconstitution de la famille-communauté se fait sous l'impulsion de facteurs contemporains : l'augmentation de l'espérance de vie et du pouvoir d'achat des retraités. Comme à propos de l'économie paysanne, on retrouve ici une sorte d'ironie de l'histoire au sens hégélien. C'est-à-dire que les développements socioéconomiques les plus contemporains : moyennisation de la société, augmentation de l'espérance de vie, libéralisation des mœurs rendent possible un retour à un modèle antérieur de communautés rurales centrées sur la parenté. Toutefois, ici encore, Mendras prend soin de faire le tri entre ce qu'on reprend et ce qu'on laisse. Ainsi, cette communauté lui parait essentielle pour l'épanouissement des individus, mais il en écarte l'aspect clos et subi. La refonte de ces communautés familiales est toujours libre : le petit-fils Astruc, élevé en ville, veut revenir à la campagne, se faire chevrier ${ }^{60}$. La grand-mère, la mère et la fille Gellenave choisissent ensemble que la fille ira chez sa grand-mère ${ }^{61}$. La famille se trouve donc érigée en communauté d'élection, voire en communauté politique ${ }^{62}$.

Ce schéma tend à naturaliser la communauté familiale mais sans lui donner de définition stricte : les liens de parenté peuvent être plus ou moins distants, les unions plus ou moins libres. L'essentiel semble être la reconnaissance d'une identité commune spontanée ou prépolitique au sens d'Habermas ${ }^{63}$, paradoxalement rendue possible par l'importance politique accordée à son existence en tant que communauté ${ }^{64}$. C'est là le nœud du système utopique de Mendras. Sorte de fait social total, moment où la société prend ${ }^{65}$,

59. Ibid., p. 45 et 97.

60. Ibid., p. 66.

61. Ibid., p. 172.

62. Ibid., p. 114 : dans le PUR, le pouvoir administratif est divisé au profit des petites communautés locales et donc des familles lorsqu'elles sont regroupées géographiquement.

63. Jürgen Habermas, Entre naturalisme et religion : les défis de la démocratie, Gallimard, Paris, 2008, p. 157.

64. Henri Mendras, op. cit., p. 100.

65. Marcel Mauss, Essai sur le don, PUF, Paris, 2012 ; et aussi Bruno Karsenti, Marcel Mauss : le fait social total, PUF, Paris, 1994. 
la manière dont l'individu s'intègre dans sa famille en tant que lieu (au sens propre et figuré) détermine le niveau d'harmonie de sa vie. La famille est ici l'équivalent social du pays. Utiliser le terme « pays » plutôt que « région », utiliser le terme « famille» plutôt que « communauté » tend à charger ces termes d'une dimension organique et sensuelle, par opposition à des termes trop froidement rationnels. Conformément au modèle romantique de réconciliation entre nature et culture, la famille et le pays sont, dans l'utopie de Mendras, naturalisés et posés comme les conditions de la culture authentique.

\section{Communauté naturelle et liberté individuelle}

On retrouve à ce point l'utopie blochienne, qui déploie une dialectique avec la nature, lieu indépassable et donc nécessaire de la liberté humaine. Pour Bloch, la nature doit devenir Foyer (Heimat) dans sa transformation en « lieu juste, où elle s'épanouit, désaliénée, en tant que bien médiatisé66 ${ }^{6}$. Ici, la désaliénation de la nature permet la désaliénation de l'homme. De même, dans l'utopie rustique la famille est naturalisée en tant que structure mais déconstruite et recomposée dans différentes formes. L'homme se libère en acceptant et en créant via la nature. Considérer la famille comme une structure naturelle revient alors à concevoir sa « coproductivité $^{67} »$, c'est-à-dire sa nécessité dans le déploiement de la liberté. La manière dont Herder démontre le besoin de chacun de s'épanouir dans son être propre, géographiquement et historiquement situé, nourrit aussi cette réflexion. Selon lui, chacun « porte en lui-même son centre de félicité ${ }^{8}{ } »$ et doit se plonger dans les traits les plus particuliers de son appartenance au monde (communauté, géographie, histoire) pour accéder à l'universel ${ }^{69}$. Dans cet ordre d'idée, l'expression de l'individualité la plus harmonieuse est en fait rendue possible par un langage et une situation particulière qui

66. Ernst Bloch, Principe espérance, t. II, Gallimard, Paris, 1976, p. 296.

67. Ibid., p. 295.

68. Johann Gottfried von Herder, Une autre philosophie de l'histoire, Montaigne, Paris, 1964, p. 191.

69. Ibid., p. 169. 
sont socialement transmis : le logos est un topos $^{70}$. Pour Herder et Mendras, la famille est donc l'interface la plus adéquate ente le monde et l'individu : permettant de saisir le monde d'un côté, et de faire ses propres choix, de l'autre.

\section{Conclusion : une utopie écologiste... et chrétienne ?}

La clé de l'idéal développé au PUR repose sur la réconciliation entre culture et nature. Cette réconciliation, si elle s'inscrit dans la lignée du romantisme progressiste d'un William Morris, s'effectue de façon originale puisqu'elle prend directement en compte le contexte français des années 1970. Le niveau de richesse de la France y est une condition nécessaire, détournée au profit de l'utopie selon un schéma enthousiaste et non violent de volontarisme politique et social. Aussi, le communisme y est mis à distance en tant qu'idéal dépassé, contrepied du capitalisme qui souffre finalement des mêmes limites en pensant l'économie comme un «système autonome, dont le fonctionnement serait régi par des lois propres, indépendantes des autres relations sociales ${ }^{71} »$. Toutefois, un certain nombre de thèmes importants de l'écologie politique de l'époque ne se retrouvent pas dans ce voyage : le rapport à la technique, par exemple.

Cette utopie s'inscrit également dans un imaginaire de la « troisième voie » fortement lié à un discours chrétien ${ }^{72}$. L'opposition entre économie paysanne et capitalisme ou communisme rejoint une vision non conformiste et personnaliste des années 1930-1940 ${ }^{73}$. Là, comme chez Mendras, la Révolution est une révolution des consciences, une bataille morale - la substitution de la qualité à la quantité, de l'être à l'avoir -, avant d'être un renversement des rapports de production. Dans son livre, Mendras évoque l'importance

70. Guillaume Lavallée, « Herder et le romantisme politique », vol. II, Phares, Laval, 2001.

71. Cornélius Castoriadis, L'Institution imaginaire de la société, Seuil, Paris, 1975, p. 25.

72. Notamment au sein du mouvement «Économie et humanisme » : Jean-Marie Gatheron, Le Pain et l'or, Économie et humanisme, Écully, 1944. 2000 .

73. Voir par exemple Emmanuel Mounier, Refaire la Renaissance, Seuil, Paris, 
de la Jeunesse agricole chrétienne (JAC) ${ }^{74}$ et parle des maisons familiales d'apprentissage rural, d'inspiration catholique, en tant que précurseurs de la pédagogie du PUR ${ }^{75}$. Enfin, il fait état de «l'effervescence religieuse » du PUR, et sous-entend le lien direct entre l'acronyme PUR et le catharisme (qui vient du grec katharos, c'est-à-dire « pur »), affirmant ainsi l'importance culturelle, voire folklorique, de la spiritualité ${ }^{76}$ dans la construction des identités.

Finalement, la façon dont Mendras s'inscrit dans ces différentes lignées de l'imaginaire utopique permet de concilier l'opposition évoquée en introduction entre utopie subversive et utopie complaisante. L'hypothèse centrale de cette utopie réside dans l'idée d'une continuité entre changements sociaux liés au modèle capitaliste occidentale - où règne la recherche de la quantité - et avènement du PUR - où règne la qualité de la vie. Il y a donc bien une complaisance pour le système tel qu'il va, qui permet l'utopie. Mais il y a également subversion, au sens étymologique de retournement, de cet ordre des choses : l'idée que son détournement est simple et joyeux. On a certes là une utopie élitiste, destinée aux classes moyennes intellectuelles, mais de l'aveu même de Mendras elle n'a d'autre ambition que de faire vivre, déjà dans l'imaginaire, la possibilité de choisir ${ }^{77}$. Ainsi, c'est bien sur le plan de l'imaginaire, d'un imaginaire lucide et honnête mais aussi romantique et exigeant, que cette utopie engage la lutte en prenant le paysan comme héraut.

74. Henri Mendras, op. cit., p. 63.

75. Ibid., p. 50.

76. Ibid., p. 143-144.

77. Ibid., p. 191. 